Leonard H. Calabrese, DO Department of Rheumatic and Immunologic| Diseases, Orthopedic \& Rheumatologic Institute, Cleveland Clinic; Professor, Cleveland Clinic Lerner College of Medicine of Case Western Reserve University, Cleveland, $\mathrm{OH}$
Cassandra Calabrese, DO

\title{
Baricitinib and dexamethasone for hospitalized patients with COVID-19
}

\section{Posted January 26, 2021}

\section{ABSTRACT}

The glucocorticoid dexamethasone is the standard of care in critically ill patients with COVID-19 to suppress the inappropriately heightened inflammatory response (cytokine storm), but the Janus kinase inhibitor baricitinib combined with remdesivir has received emergency use authorization for the same indication. As of this writing, in a hospitalized patient with COVID-19 who has evidence of pneumonitis or hypoxia, we recommend using either regimen, but not both together. Both regimens have shown benefit in randomized controlled trials, but we cannot state with certainty that either is superior to the other, nor whether they should be used together. Further trials are underway.

\section{INTRODUCTION}

Mortality rates in patients with COVID-19 have fallen dramatically since the beginning of the pandemic, especially among hospitalized patients in intensive care units. ${ }^{1}$ Several factors may account for this trend, one being more aggressive and effective management of the third (hyperinflammatory) phase of advanced COVID-19. ${ }^{2}$ Use of dexamethasone has now become a standard of care for hospitalized patients requiring oxygen.

Literally hundreds of studies are underway investigating a myriad of therapeutics including biologics, targeted synthetic therapies, traditional immunosuppressives, and repurposed drugs, but thus far, the vast majority of studies have been negative or showed modest effects. We have reviewed several leading candidate therapies in these pages over the past several months. ${ }^{3-5}$

The statements and opinions expressed in COVID-19 Curbside Consults are based on experience and the available literature as of the date posted. While we try to regularly update this content, any offered recommendations cannot be substituted for the clinical judgment of clinicians caring for individual patients.

doi:10.3949/ccjm.88a.ccc073
Since the publication of our recent review of Janus kinase (JAK) inhibitors in COVID-19 disease, ${ }^{5}$ the JAK inhibitor baricitnib, in combination with remdesivir, has been granted emergency use authorization by the US Food and Drug Administration ${ }^{6}$ to treat suspected or laboratory-confirmed COVID-19 in hospitalized adults and pediatric patients at least 2 years of age requiring supplemental oxygen, invasive mechanical ventilation, or extracorporeal membrane oxygenation. The recommended dose for adults is $4 \mathrm{mg}$ per day for 14 days, with dose adjustments for renal or hepatic impairment. ${ }^{6}$

\section{ACTT-2: MODEST BENEFIT FROM BARICITINIB}

The basis for this approval was the second Adaptive Covid-19 Treatment Trial (ACTT-2), ${ }^{7}$ which was a randomized double-blind controlled trial evaluating baricitinib plus remdesivir vs remdesivir alone in hospitalized patients with COVID-19 and evidence of pneumonia or pulmonary compromise, conducted in 1,033 patients and ultimately published on December 11,2020 . The primary endpoint for the intention-totreat population was time to recovery within 29 days after randomization. The key secondary endpoint was clinical status on day 15, assessed on an 8-point ordinal scale. Prior or concomitant immunosuppressive therapies were prohibited unless the hospital had a written protocol for such. Glucocorticoids were explicitly prohibited unless indicated for medical reasons (eg, allergy, asthma, laryngeal edema).

Overall, the effects of this combined regimen were modest, with a 1-day shortening of recovery timemedian 7 days (95\% confidence interval [CI] 6-8) vs 8 days (95\% CI 7-9). The 28-day mortality rate was $5.1 \%$ in the active treatment group vs $7.8 \%$ in the control group (hazard ratio [HR] 0.65, 95\% CI 0.391.09). The effect size was greatest for those requiring noninvasive ventilation or high-flow oxygen and 
lowest for those who did not need oxygen, suggesting that stage and timing of treatment may be critical, though none of these secondary outcomes achieved statistical significance.

Serious adverse events were less frequent in the active treatment group, and in particular there was no increase in thromboembolic events or serious infections which, as noted in our previous review, has been a concern with JAK inhibitors, which carry a class warning for such in their labeling for treatment of other conditions. ${ }^{5}$

\section{RECOVERY TRIAL: BENEFIT OF DEXAMETHASONE}

What remains to be determined is how this newly authorized therapy measures up to dexamethasone, which is now a standard of care for COVID-19 based on the robust Randomized Evaluation of Covid-19 Therapy (RECOVERY) trial. ${ }^{8}$

This study, which involved 6,425 patients, demonstrated the efficacy of dexamethasone (without antivirals) in hospitalized patients with COVID-19. The primary endpoint was mortality at 28 days.

The mortality rate was $22.9 \%$ in the active treatment group and $25.7 \%$ in the control group $(95 \% \mathrm{CI}$ $0.75-0.93 P<.001)$. The effect size was most robust in those requiring mechanical ventilation, with no discernable effect in those not requiring oxygen and with trends for harm in that group, suggesting the critical importance of timing and stage of disease.

Comparing these 2 studies is problematic for a variety of reasons. First, the RECOVERY trial was conducted a little earlier, commencing enrollment on March 19th, while ACTT-2 began enrolling on May 8th, and during this interval meaningful strides were made in critical care management. ${ }^{1}$ Evidence of this is the dramatic difference in the mortality rates in the control groups of the 2 trials $(25.7 \%$ in the RECOVERY trial vs $7.8 \%$ in ACTT-2), strongly suggesting these are not comparable populations. In addition, none of the patients in RECOVERY were treated with baricitinib, but 223 patients in ACTT-2 received glucocorticoids for varying medical indications.

\section{NIH GUIDELINES}

Thus, a practical question at this moment is where do we stand in terms of managing hospitalized patients with COVID-19 complicated by pneumonitis, hypoxia, or both using dexamethasone, baricitinib, or both? Guidelines from the National Institutes of Health, ${ }^{9}$ issued December 14, 2020, suggest the following:
- "There are insufficient data for the Panel to recommend either for or against the use of baricitinib in combination with remdesivir for the treatment of COVID-19 in hospitalized patients in cases where corticosteroids can be used instead.

- In the rare circumstances where corticosteroids cannot be used, the Panel recommends using baricitinib in combination with remdesivir for the treatment of COVID-19 in hospitalized, nonintubated patients who require oxygen supplementation.

- The Panel recommends against the use of baricitinib in the absence of remdesivir, except in a clinical trial.

- There are insufficient data for the Panel to recommend either for or against the use of baricitinib in combination with corticosteroids for the treatment of COVID-19. Since both agents are potent immunosuppressants, there is potential for an additive risk of infection."

As of this writing, the Infectious Disease Society of America has yet to articulate a formal position on the use of baricitinib, but one will likely soon be forthcoming.

\section{SUPPRESSING HYPERINFLAMMATION}

It is important to note that both of these drugs are well poised, via their known mechanisms of action, to suppress in the hyperinflammatory phase of COVID19 (Figure 1). ${ }^{2}$ Dexamethasone, a long-acting glucocorticoid, binds to glucocorticoid receptors, which are ligand-dependent transcription factors. Liganded glucocorticoid receptors in turn bind to glucocorticoid-responsive elements in target genes, thus influencing a host of cellular processes. These effects can be broadly immunosuppressive including inhibition elements of the interferon response as well as B cells, $\mathrm{T}$ cells, and antigen-presenting cells. ${ }^{10}$ Glucocorticoids also have a well-recognized and dose-dependent adverse effect profile. ${ }^{11}$

Baricitinib, a selective inhibitor of Janus kinase signal transduction, modulates the biologic activity of a broad array of inflammatory cytokines. On the other hand, it has countervailing effects by also inhibiting the signal transduction of type 1 and type 3 interferons, which are important early in the antiviral host response. Thus, the drug has a formidable toxicity profile including serious infections, viral reactivation (ie, zoster) and venous thrombotic complications. ${ }^{4,5}$

We know nearly nothing about combining these drugs in the setting of COVD-19 but would postulate 


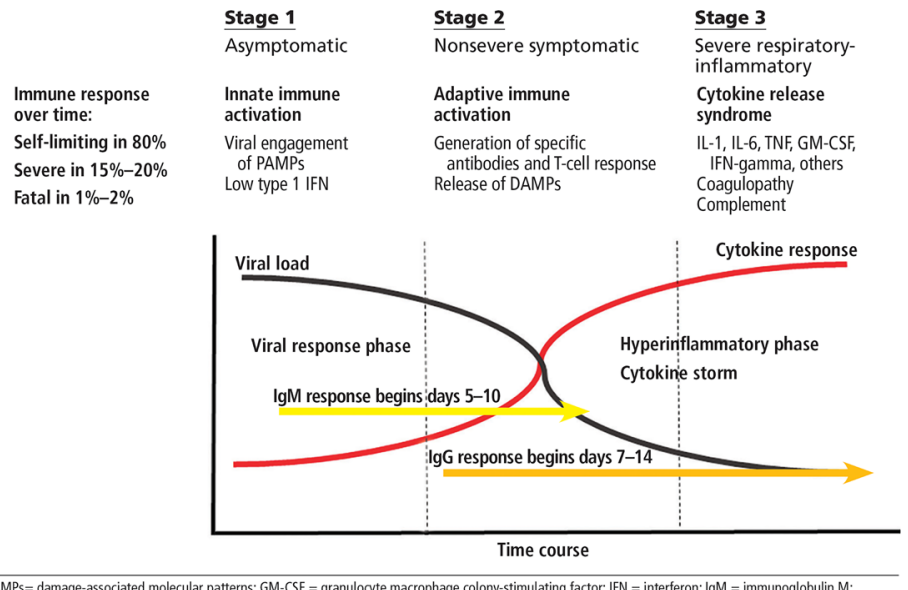

DAMPs $=$ damage-associated molecular patterns; GM-CSF = granulocyte macrophage colony-stimulating factor; IFN $=$ int
$\mathrm{L}-1=$ interleukin $1 ; \mathrm{L}-6$ = interleukin 6 ; PAMPs $=$ pathogen-associated molecular patterns; $\mathrm{TNF}=$ tumor necrosis factor

Figure 1. Three stages of COVID-19 disease.

Reprint from reference 2 .

that the effects would be enhanced immunosuppression and the likelihood of enhanced toxicity.

\section{TRIAL UNDERWAY}

The only way ahead is to directly compare these 2 therapies, and fortunately a major study is now underway with the Adaptive COVID-19 Treatment Trial 4 (ACTT-4; NCT04640168) sponsored by the National Institute of Allergy and Infective Diseases as part of the Adaptive COVID-19 Treatment Trial initiative. This trial will recruit 1,500 patients, with the primary objective of evaluating the clinical efficacy of baricitinib plus remdesivir vs dexamethasone plus remdesivir as assessed by the mechanical ventilationfree survival by day 29. The key secondary objective is to evaluate the clinical efficacy of baricitinib plus remdesivir vs dexamethasone plus remdesivir according to clinical status (8-point ordinal scale) at day 15 .

\section{WHAT DO WE KNOW NOW?}

For the moment, we believe that when confronted by a hospitalized patient with COVID-19 with evidence of pneumonitis or hypoxia, it prudent to utilize one or the other of these regimens until trials such as ACTT-4 are completed. At the moment we have equipoise to support the use of either agent and believe the choice may be more influenced by concerns over toxicities from comorbidities (eg, diabetes and neuromuscular weakness with glucocorticoids vs active immunothrombosis for baricitinib). We do not support empirically combining these agents in the setting of COVID-19, owing to concerns of excess immunosuppression.

\section{DISCLOSURES}

Dr. Leonard Calabrese has disclosed financial relationships (consulting, teaching, or speaking) with Abbvie Pharmaceuticals, BMS, Crescendo, GSK, Genentech-Roche, Horizon Pharma, Janssen, Novartis, Pfizer, Regeneron, Sanofi Aventis, and USB. Dr. Cassandra Calabrese has disclosed financial relationships (consulting, teaching, or speaking) with Abbvie and Sanofi-Regeneron.

\section{REFERENCES}

1. Ledford H. News feature. Why do COVID death rates seem to be falling? Nature November 11, 2020. Accessed January 19, 2021. https://www.nature.com/articles/d41586-020-03132-4

2. Calabrese LH. Cytokine storm and the prospects for immunotherapy with COVID-19. Cleve Clin J Med 2020; 87(7):389-393. doi:10.3949/ ccjm.87a.ccc008

3. Calabrese C, Rajendram P, Sacha GL, Calabrese L. Practical aspects of targeting IL-6 in COVID-19 disease. Cleve Clin J Med 2020. doi: 10.3949/ccjm.87a.ccc018

4. Calabrese LH, Lenfant T, Calabrese C. Cytokine storm release syndrome and the prospects for immunotherapy with COVID-19, part 3: the role of GM-CSF. Cleve Clin J Med 2020. doi:10.3949/ccjm.87a. ccc057

5. Calabrese LH, Lenfant T, Calabrese C. Cytokine storm release syndrome and the prospects for immunotherapy with COVID-19, part 4: the role of JAK inhibition. Cleve Clin J Med 2020. doi:10.3949/ ccjm.87a.ccc060

6. US Food and Drug Administration. Fact sheet for healthcare providers. Emergency use authorization (EUA) of baricitinib. November 19, 2020. Accessed January 19, 2021. https://www.fda.gov/ media/143823/download (November 19th, 2020)

7. Kalil AC, Patterson TF, Mehta AK, et al for the ACTT-2 Study Group Members. Baricitinib plus remdesivir for hospitalized adults with Covid-19. N Engl J Med 2020 Dec 11. doi:10.1056/NEJMoa2031994

8. The RECOVERY Collaborative Group. Dexamethasone in hospitalized patients with Covid-19 - preliminary report. N Engl J Med 2020 Jul 17. doi:10.1056/NEJMoa2021436

9. National Institutes of Health. The COVID-19 Treatment Guidelines Panel's statement on the emergency use authorization of baricitinib for the treatment of COVID-19. November 14, 2020. Accessed January 19, 2021. https://www.covid19treatmentguidelines.nih. gov/statement-on-baricitinib-eua/

10. Cain DW, Cidlowski JA. After 62 years of regulating immunity, dexamethasone meets COVID-19. Nature Rev Immunol 2020; 20(10):587-588. doi:10.1038/s41577-020-00421-x

11. Ahmed $\mathbf{M H}$, Hassan A. Dexamethasone for the treatment of coronavirus disease (COVID-19): a review. SN Compr Clin Med 2020 Oct 31:1-10. doi:10.1007/s42399-020-00610-8

Correspondence: Leonard H. Calabrese, DO, Department of Rheumatic and Immunologic Diseases, A50, Cleveland Clinic, 9500 Euclid Avenue, Cleveland, OH 44195; calabrl@ccf.org 